Research Article

\title{
The Influence of Initial Normal Stress Assumption of Slip Surface on Safety Factor of Symmetrical Three-Dimensional Slopes
}

\author{
Huali Liu \\ Army Engineering University of PLA, Nanjing 210007, China \\ Correspondence should be addressed to Huali Liu; 1429211066@qq.com
}

Received 23 December 2018; Accepted 14 April 2019; Published 15 May 2019

Academic Editor: Michele Zappalorto

Copyright (C) 2019 Huali Liu. This is an open access article distributed under the Creative Commons Attribution License, which permits unrestricted use, distribution, and reproduction in any medium, provided the original work is properly cited.

\begin{abstract}
By using the explicit solution of three-dimensional slope stability based on modification of normal stress distribution over the slip surface, the influence of assumption of the three-dimensional initial normal stress on the safety factor is investigated. The initial normal stress distribution over the 3D slip surface was assumed, and then it was modified by a function with 2 parameters to satisfy two force equilibrium conditions about two axes and one moment equilibrium condition around one axis. An iterative equation was derived that would yield a value to $3 \mathrm{D}$ safety factor. The values of three-dimensional safety factor of symmetrical slopes are computed with different assumptions of initial normal stresses. The computation results show that the influence of assumption of initial normal stress on the safety factor of symmetrical three-dimensional slopes is negligible because the maximum different value of the three-dimensional safety factor is below $5 \%$.
\end{abstract}

\section{Introduction}

The limit equilibrium method has widely been used for slope-stability analysis. Experts consider that only rigorous limit equilibrium methods are recommended for slope-stability analysis because they satisfy both force and moment balance conditions. The 3D safety factor obtained can satisfy the engineering needs, and this result was later agreed by Duncan [1]. However, researches show that the difference of the rigorous safety factors of the limit equilibrium method is obvious, and the method may not provide a unique safety factor. In order to solve these problems, some methods have widely been studied by Ugai et al. [2,3]. These methods introducing various assumptions, especially for the threedimensional slope, cause great difference in the safety factor and failure in utilizing the limit equilibrium method in engineering practice. Researchers have also shown that the difference of safety factors is about $15 \%$ for the two-dimensional slope and reaches to $40 \%$ for three-dimensional slope, so these methods cannot directly be applied in engineering practice. Moreover, these methods cannot obtain rigorous 3D limit equilibrium solutions, and this conclusion has been proven by Zhu et al. [4]. In recent years, the study shows that the explicit solution of two-dimensional slope stability based on modification of normal stress distribution over the slip surface can be obtained satisfying both forces equilibrium conditions and moment equilibrium conditions. So, the explicit solution of three-dimensional slope stability based on modification of normal stress distribution over the slip surface can also be obtained satisfying both forces of equilibrium conditions and moment equilibrium conditions. These methods are not divided into slices or columns and have been studied by Zhu et al. [5]. By using the explicit solution of threedimensional slope stability based on modification of normal stress distribution over the slip surface, the influence of assumption of the three-dimensional initial normal stress on the factor safety is investigated. The initial normal stress distribution over the 3D slip surface was assumed, and then it was modified by a function with 


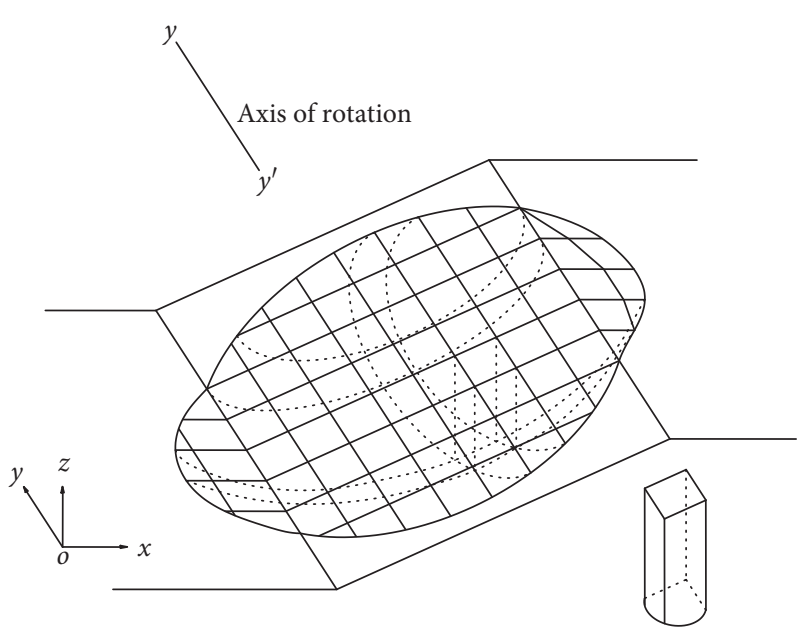

Figure 1: The 3D slip surface and coordinate.

2 parameters to satisfy two force equilibrium conditions about two axes and one moment equilibrium condition around one axis. An iterative equation was derived to yield a value to $3 \mathrm{D}$ factor safety. The values of symmetrical three-dimensional safety factor are computed with different assumptions of initial normal stress. The influence of assumption of the three-dimensional initial normal stress on the safety factor is the key to problem. It cannot be directly applied to engineering if the difference of $3 \mathrm{D}$ safety factor is more than $30 \%$ and it can be directly applied in engineering if the difference of $3 \mathrm{D}$ safety factor is less than or equal to $30 \%$ in engineering practice [5].

\section{Basic Concepts}

Consider a slip surface of a $3 \mathrm{D}$ general shape, as shown in Figure 1, and the slip horizontal surface and slip surface are described by functions $g(x, y)$ and $s(x, y) . w(x, y)$ is the total weight of the column; $k_{c} w(x, y)$ is the internal force due to an earthquake, where $k_{c}$ is the coefficient of seismic force which is assumed to be horizontal; $\sigma(x, y)$ is the normal stress over the slip surface; $\tau(x, y)$ is the shear stress on the slip surface, and $u(x, y)$ is the water pressure of the slip body.

The normal force is assumed as follows:

$$
\begin{aligned}
\sigma(x, y) & =\sigma_{0}(x, y) \cdot\left[\lambda_{1} \xi_{1}(x, y)+\lambda_{2} \xi_{2}(x, y)\right] \\
& =\sigma_{0}(x, y) \cdot\left[\lambda_{1} x+\lambda_{2} y\right] .
\end{aligned}
$$

As shown in Figure 1, a rectangular coordinate system is established, above which the sliding mass is divided into $n$ small columns along the direction of the $x$-axis and divided into $m$ small columns along the $y$-axis, and then the whole sliding mass is divided into $n \times m$ columns as shown in Figure 2.

Now choose a typical column taking the $j$ th and the $i$ th columns along the $x$-axis and the $y$-axis to examine the forces acting upon it (Figure 2). It should be noted that

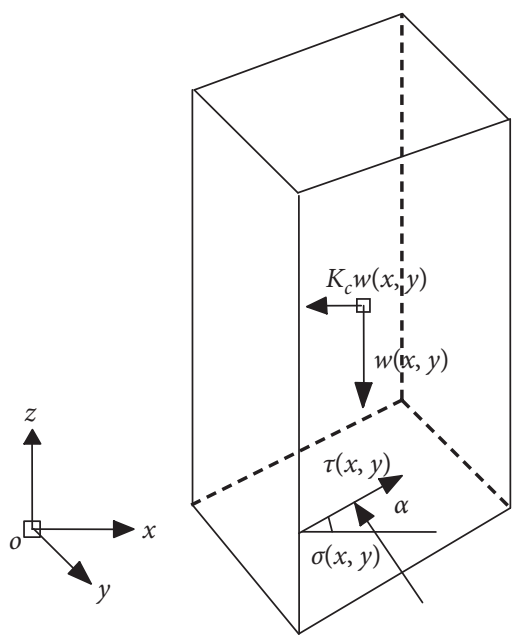

Figure 2: Forces acting on a column.

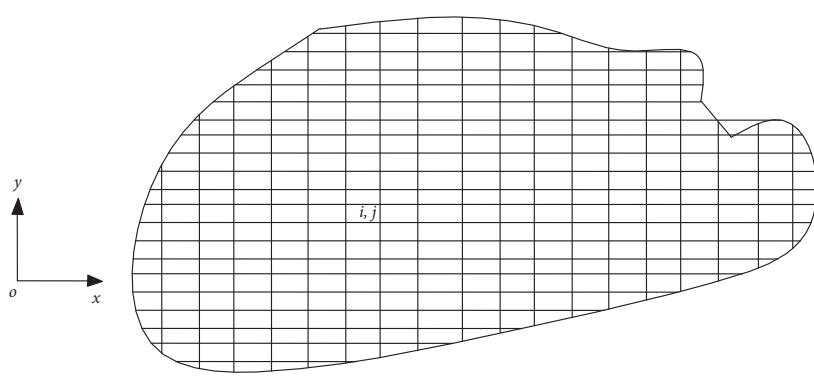

FIgURE 3: The projection of 3D failure mass in the xoy plane.

effective stress is considered in this paper, but the approach is also certainly applicable to total stress. $\left(n_{x}, n_{y}, n_{z}\right)$ is the direction cosine of the normal force $\sigma(x, y) ;\left(m_{x}, m_{y}, m_{z}\right)$ is the direction cosine of the shear force $\tau(x, y) ;\left(x_{c}, y_{c}, z_{c}\right)$ is the center point coordinate of the column; and $a$ is the inclination of the bottom of a column.

For the purposes of simplification, $(x, y)$ will be omitted in the following paper. We will get $m_{y}=0$, if sliding mass is sliding in the xoz plane but not sliding along the $y$ axis.

Since $s(x, y)$ is the slip surface, the outer normal direction of the slip surface is $((\partial s / \partial x),(\partial s / \partial y),-1)$ according to the definition of the outer normal line. The direction of the slip surface is opposite to the normal force direction of the slip surface, so the direction cosine of the slip surface normal force is

$$
\begin{aligned}
\left(n_{x}, n_{y}, n_{z}\right) & =\left(-\frac{\partial s / \partial x}{\Delta},-\frac{\partial s / \partial y}{\Delta}, \frac{1}{\Delta}\right) \\
\Delta & =\sqrt{1+\left(\frac{\partial s}{\partial x}\right)^{2}+\left(\frac{\partial s}{\partial y}\right)^{2}} .
\end{aligned}
$$

The shear direction is perpendicular to the normal force direction, so 


$$
\begin{aligned}
\left(m_{x}, m_{y}, m_{z}\right) & =\left(\frac{1}{\Delta^{\prime}}, 0, \frac{\partial s / \partial x}{\Delta^{\prime}}\right) \\
\Delta^{\prime} & =\sqrt{1+\left(\frac{\partial s}{\partial x}\right)^{2}} .
\end{aligned}
$$

The area of a rectangle is $d x d y$, and the surface area is $\mathrm{dA}$, as shown in Figure 3, when the slip surface of a small column is projected to the $x-y$ plane. Then,

$$
\mathrm{dA}=\frac{d x d y}{n_{z}}=\Delta d x d y=\sqrt{1+\left(\frac{\partial s}{\partial x}\right)^{2}+\left(\frac{\partial s}{\partial y}\right)^{2}} d x d y
$$

\section{Basic Formula}

Three equilibrium conditions only need to be considered for symmetrical three-dimensional slopes, and six equilibrium conditions need to be considered for asymmetrical threedimensional slopes. Two force equilibrium conditions on $x$ and $z$-axis and one moment equilibrium condition around $y$ axis of the sliding mass for symmetrical slopes are as follows:

$$
\begin{aligned}
& \iint\left(\sigma \cdot \mathrm{dA} \cdot n_{x}+\tau \cdot \mathrm{dA} \cdot m_{x}\right)=\iint K_{c} \cdot w(x, y) d x d y, \\
& \iint\left(\sigma \cdot \mathrm{dA} \cdot n_{z}+\tau \cdot \mathrm{dA} \cdot m_{z}\right)=\iint w(x, y) d x d y, \\
& \quad \iint \sigma \cdot \mathrm{dA} \cdot n_{x} \cdot s-\iint \tau \cdot \mathrm{dA} \cdot m_{x} \cdot s+\iint K_{c} w(x, y) \cdot z_{c} d x d y \\
& \quad+\iint \sigma \cdot \mathrm{dA} \cdot n_{z} \cdot x+\iint \tau \cdot \mathrm{dA} \cdot m_{z} \cdot x \\
& \quad-\iint w(x, y) \cdot x_{c} d x d y=0 .
\end{aligned}
$$

Substituting equations (2)-(4) into equations (5a)-(5c), we have

$$
\begin{aligned}
& -\iint \sigma \cdot \frac{\partial s}{\partial x} \cdot d x d y+\iint \tau \cdot \frac{\Delta}{\Delta^{\prime}} d x d y=\iint K_{c} \cdot w(x, y) d x d y \\
& \iint \sigma \cdot d x d y+\iint \tau \cdot \frac{\partial s}{\partial x} \cdot \frac{\Delta}{\Delta^{\prime}} d x d y=\iint w(x, y) d x d y
\end{aligned}
$$

$$
\begin{aligned}
& \iint \sigma \cdot \frac{\partial s}{\partial x} \cdot s d x d y-\iint \tau \cdot \frac{\Delta}{\Delta^{\prime}} \cdot s d x d y \\
& \quad+\iint K_{c} w(x, y) \cdot z_{c} d x d y+\iint \sigma \cdot x \cdot d x d y \\
& \quad+\iint \tau \cdot \frac{\partial s}{\partial x} \cdot \frac{\Delta}{\Delta^{\prime}} \cdot x \cdot d x d y-\iint w(x, y) \cdot x_{c} d x d y=0 .
\end{aligned}
$$

According to the Mohr-Coulomb criterion,

$$
\tau(x, y)=\frac{[\sigma(x, y)-u(x, y)] \cdot \tan \phi(x, y)+c(x, y)}{F_{s}}
$$

where $\phi(x, y)$ is the effective internal friction angle and $c(x, y)$ is the cohesion of the sliding mass. We may use " $\psi$ " to express $\tan \phi(x, y)$. have

Substituting equation (7) into equations (6a)-(6c), we

$$
\begin{gathered}
\iint\left[-\frac{\partial s}{\partial x}+\frac{\Delta}{\Delta^{\prime}} \frac{\psi}{F_{s}}\right] \sigma \cdot d x d y=\iint K_{c} w d x d y \\
+\iint \frac{u \cdot \psi-c}{F_{s}} \frac{\Delta}{\Delta^{\prime}} d x d y, \\
\iint\left[1+\frac{\partial s}{\partial x} \cdot \frac{\Delta}{\Delta^{\prime}} \frac{\psi}{F_{s}}\right] \sigma \cdot d x d y=\iint w d x d y \\
+\iint \frac{u \cdot \psi-c}{F_{s}} \frac{\partial s}{\partial x} \cdot \frac{\Delta}{\Delta^{\prime}} d x d y, \\
\iint\left[\frac{\partial s}{\partial x} \cdot s-\frac{\psi \cdot s}{F_{s}} \frac{\Delta}{\Delta^{\prime}}+x+\frac{\partial s}{\partial x} \frac{\Delta}{\Delta^{\prime}} \frac{\psi}{F_{s}} \cdot x\right] \sigma \cdot d x d y \\
=-\iint K_{c} w \cdot z_{c} d x d y+\iint w \cdot x_{c} d x d y \\
\quad-\iint \frac{u \cdot \psi-c}{F_{s}} \cdot \frac{\Delta}{\Delta^{\prime}} s d x d y+\iint \frac{u \cdot \psi-c}{F_{s}} \cdot \frac{\partial s}{\partial x} \\
\quad \cdot \frac{\Delta}{\Delta^{\prime}} \cdot x d x d y \cdot
\end{gathered}
$$

Supposing $\quad F_{x}=\iint K_{c} \cdot w d x d y ; \quad F_{y}=\iint w d x d y ;$ $r_{\sigma}=x+s \cdot s_{x}^{\prime} ; \quad r_{\tau}=s_{x}^{\prime} \cdot x-s, \quad M_{c}=\iint w \cdot x_{c} d x d y-\iint K_{c}$ $\cdot w \cdot z_{c} d x d y$; and $\Delta / \Delta^{\prime}=\rho$.

Substituting equation (1) into equations (8a)-(8c), we have

$$
\begin{aligned}
& \lambda_{1} \iint\left(-s_{x}^{\prime}+\rho \cdot \psi \frac{1}{F_{s}}\right) \cdot \xi_{1} \cdot \sigma_{0} \cdot d x d y+\lambda_{2} \iint\left(-s_{x}^{\prime}+\rho \cdot \psi \frac{1}{F_{s}}\right) \cdot \xi_{2} \cdot \sigma_{0} d x d y=F_{x}+\frac{1}{F_{s}} \iint \rho(u \cdot \psi-c) d x d y, \\
& \lambda_{1} \iint\left(1+s_{x}^{\prime} \cdot \rho \cdot \psi \frac{1}{F_{s}}\right) \cdot \xi_{1} \cdot \sigma_{0} \cdot d x d y+\lambda_{2} \iint\left(1+s_{x}^{\prime} \cdot \rho \cdot \psi \frac{1}{F_{s}}\right) \cdot \xi_{2} d x d y=F_{y}+\frac{1}{F_{s}} \iint s_{x}^{\prime} \cdot \rho(u \cdot \psi-c) d x d y,
\end{aligned}
$$




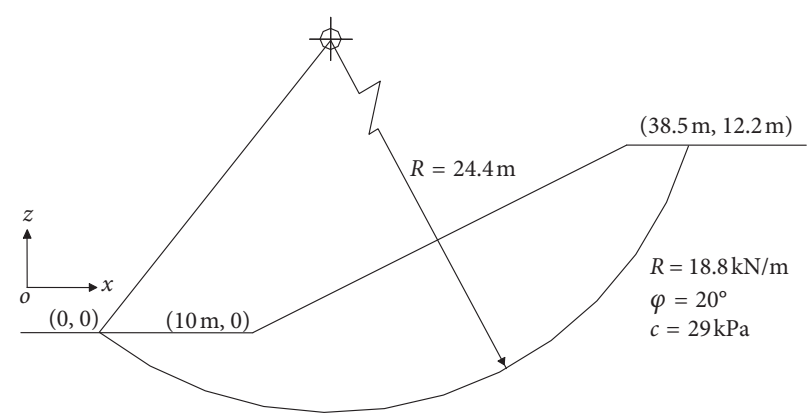

FIGURE 4: Cross section and parameters of a slope.

TABLE 1: The comparison of the calculated safety factor for cases.

\begin{tabular}{lcc}
\hline Methods & Case 1 & Case 2 \\
\hline Two-dimensional method & 2.038 & 1.003 \\
Methods in this paper & 2.305 & 1.255 \\
$\quad \sigma_{0}^{1}$ & 2.2965 & 1.254 \\
$\sigma_{0}^{2}$ & 2.322 & 1.261 \\
$\sigma_{0}^{3}$ & 2.187 & 1.401 \\
Limit equilibrium solution & 2.122 & 1.401 \\
The result computed by Hungr [9] & & 1.402 \\
\hline The result computed by Zhang [8] & 1.250 \\
The result computed by Leshchinsky [10] & \\
The result computed by Baligh and Azzouz [11] & \\
The result computed by Gens et al. [12] & & 1.402 \\
\hline
\end{tabular}

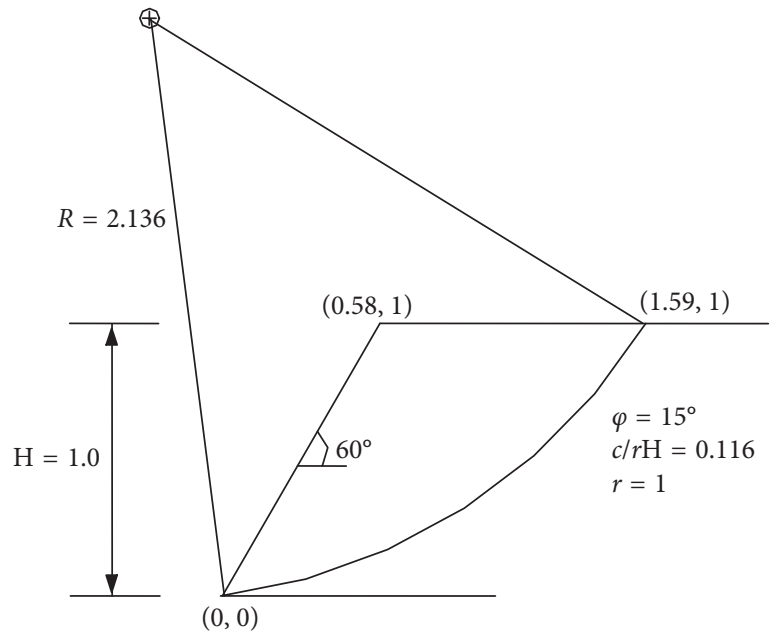

Figure 5: An ellipsoidal slip surface and parameters of a slope.

$$
F_{s}=\frac{\lambda_{1} \iint \sigma_{0} \cdot \psi \cdot \xi_{1} \cdot r_{\tau} \rho d x d y+\lambda_{2} \iint \sigma_{0} \cdot \psi \cdot \xi_{2} \cdot r_{\tau} \rho d x d y+\iint(-u \cdot \psi+c) \cdot r_{\tau} \cdot \rho d x d y}{M_{c}-\lambda_{1} \iint \sigma_{0} \cdot \xi_{1} \cdot r_{\sigma} d x d y-\lambda_{2} \iint \sigma_{0} \cdot \xi_{2} \cdot r_{\sigma} d x d y}
$$




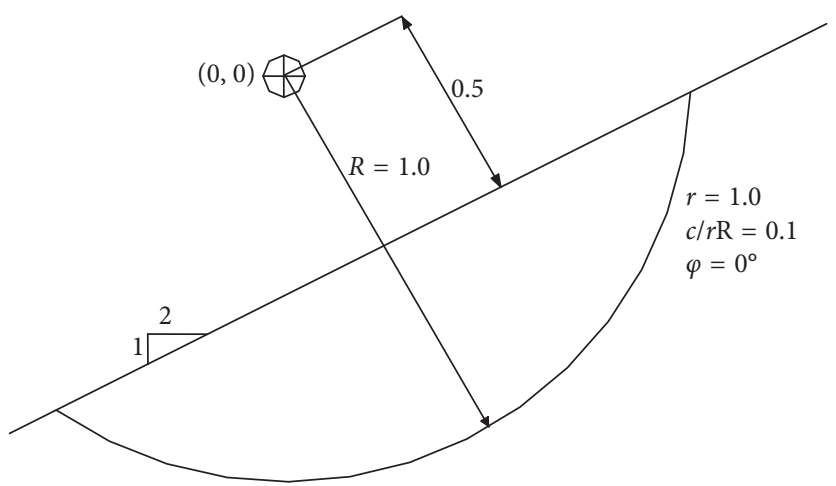

FIGURE 6: A spherical slip surface and parameters of a slope.

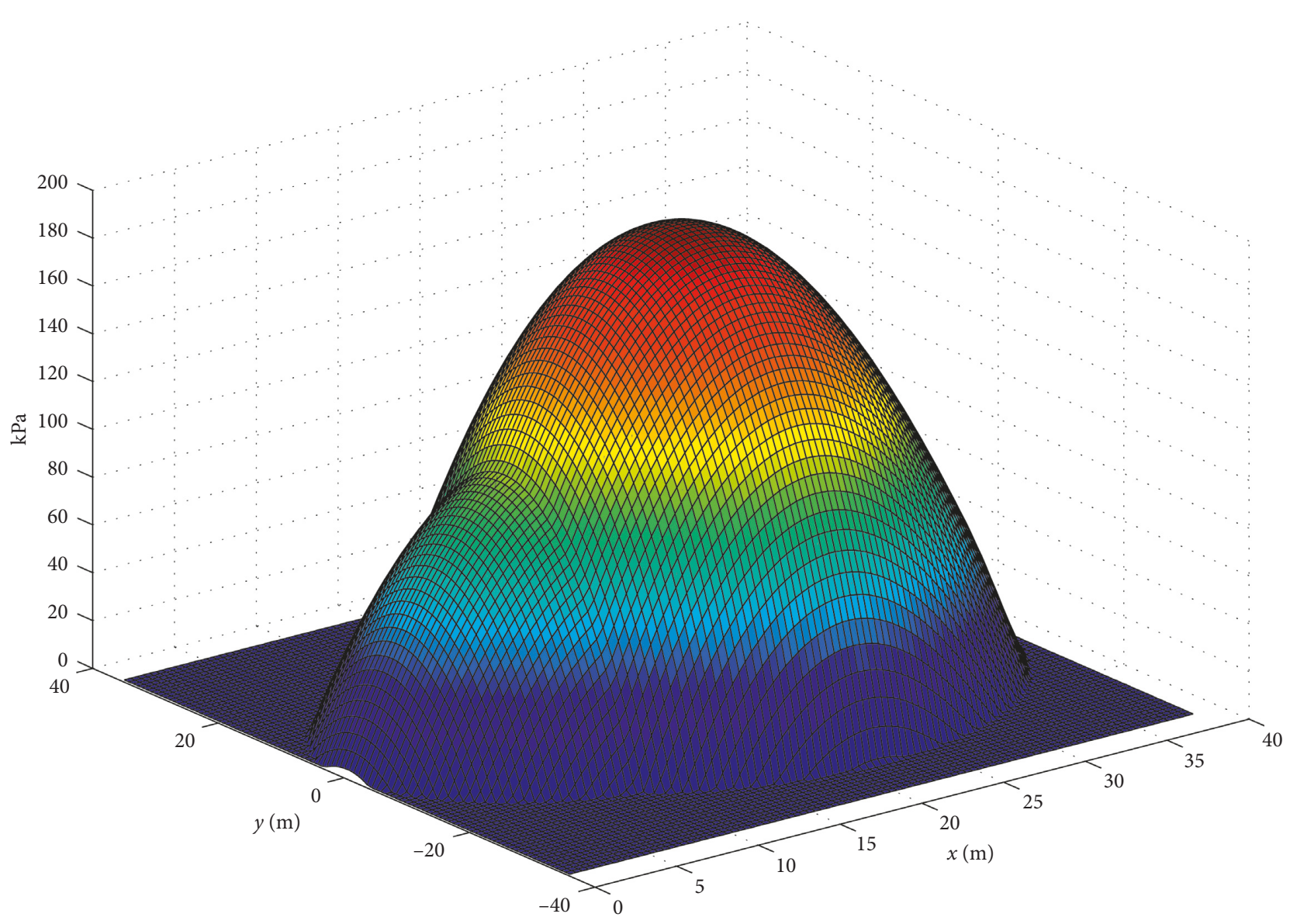

FIgURE 7: The distribution of normal stresses for case 1 .

Equations (9a)-(9c) are simplified, and then we have

$$
\begin{gathered}
\lambda_{1} \cdot\left(A_{1}+\frac{1}{F_{s}} A_{1}^{\prime}\right)+\lambda_{2} \cdot\left(A_{2}+\frac{1}{F_{s}} A_{2}^{\prime}\right)=A_{3}+\frac{1}{F_{s}} A_{3}^{\prime}, \\
\lambda_{1} \cdot\left(B_{1}+\frac{1}{F_{s}} B_{1}^{\prime}\right)+\lambda_{2} \cdot\left(B_{2}+\frac{1}{F_{s}} B_{2}^{\prime}\right)=B_{3}+\frac{1}{F_{s}} B_{3}^{\prime}, \\
F_{s}=\frac{D_{1} \lambda_{1}+D_{2} \lambda_{2}+D_{3}}{E_{1} \lambda_{1}+E_{2} \lambda_{2}+E_{3}} .
\end{gathered}
$$

The parameters are one-to-one correspondence in equations (9a)-(9c) and (10a)-(10c). Equations (10a)-(10c) are nonlinear equations containing 3 variables. Thus, an iterative procedure is required for the safety factor of the three-dimensional slope.

\section{Assumption of Initial Normal Stress over Slip Surface}

There are three kinds of $\sigma_{0}(x, y)$ hypotheses in this paper

(1) $\sigma_{0}$ is assumed as gravity stress of a column, and we have 


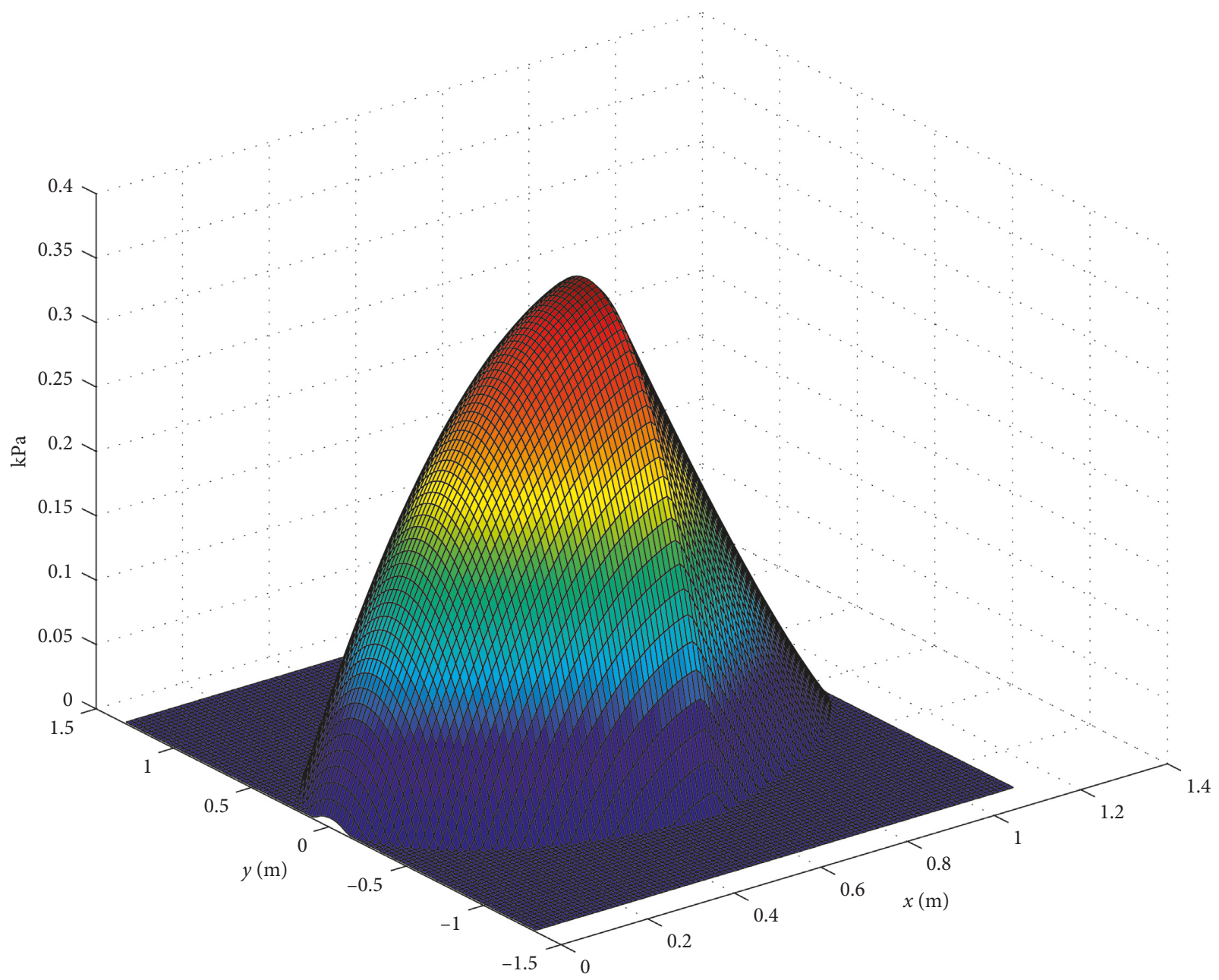

FIGURE 8: The distribution of normal stresses for case 2.

$$
\sigma_{0}^{1}=w
$$

(2) $\sigma_{0}$ is assumed as follows:

$$
\sigma_{0}^{2}=w \cdot \cos \alpha
$$

This is the extension of the Swedish method, and this method has been studied by Fellenius [6], in which the internal forces of columns are ignored.

(3) $\sigma_{0}$ is assumed to be 1 , which is the extension of the simplified Bishop method, and this method has been studied by Bishop [7]. That is to say, the internal forces of columns are horizontal, and we have

$$
\sigma_{0}^{3}=\frac{\left(w-c A \sin \alpha_{x} / F_{s}\right)+\left(u A \tan \varphi \sin \alpha_{x} / F_{s}\right)}{m_{a}} .
$$

By substituting equations (11)-(13) into equations (10a)-(10c), different 3D safety factor can be obtained.

\section{Applications}

Case 1. The example shown in Figure 4 is a typical threedimensional slope in reference, and this example has been studied by Zhang [8]. The spherical profile and soil stratigraphy are analyzed as shown in Figure 4 . The soil properties are given in Figure 4. Different symmetrical three-dimensional safety factors are presented in Table 1.

Case 2. The example shown in Figure 5 is a typical threedimensional slope in reference, and this example has been studied by Leshchinsky et al. [10]. The sliding mass is a critical ellipse (the aspect ratio is 0.66). The ellipsoidal profile and soil stratigraphy are analyzed as shown in Figure 5. The soil properties are given in Figure 5. Different symmetrical three-dimensional safety factors are presented in Table 1.

Case 3. The example shown in Figure 6 is a typical threedimensional slope in reference, and this example has been 


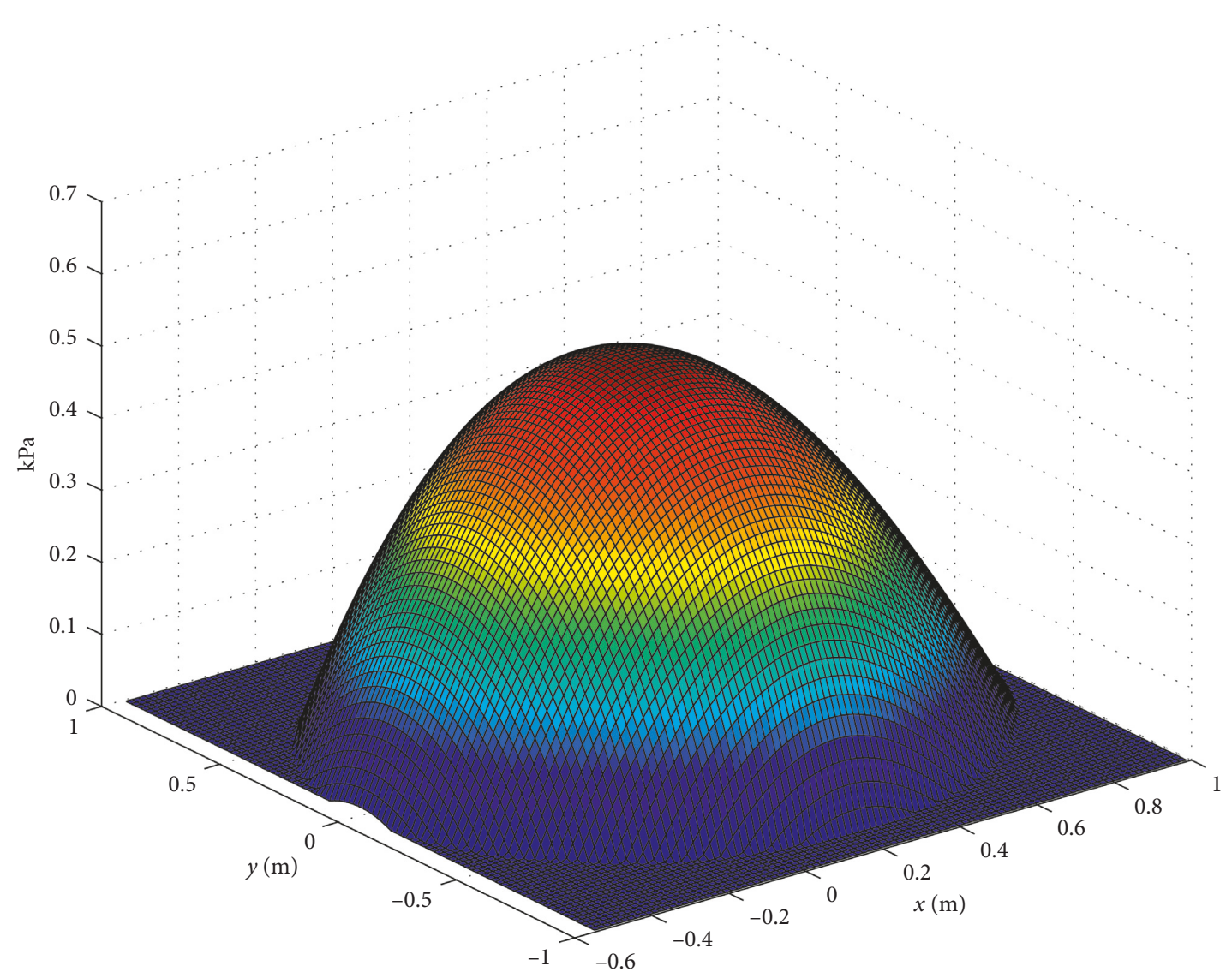

FIgURE 9: The distribution of normal stresses for case 3.

studied by Baligh and Azzouz [11]. Its frictional force is zero. The spherical profile and soil stratigraphy are analyzed as shown in Figure 6. The soil properties are given in Figure 6. Different symmetrical three-dimensional safety factors are presented in Table 1.

A comparison of values of safety factor and the associated scaling factors computed with the method proposed in this paper are presented in Table 1. The differences in the computed values of safety factor are negligible for practical purposes because the maximum different value of threedimensional safety factors is below $5 \%$. So the explicit solution of three-dimensional slope stability can be used to engineering practice.

\section{Verification of Normal Stress over Slip Surface}

Figures 7-9 are the normal stress over slip surface of case 1, case 2, and case 3. It can be seen from the figures that the normal stresses over the slip surface are positive and smooth and continuous. Therefore, they are reasonable.

\section{Concluding Remarks}

By assuming the distribution of the normal stress along the slip surface, the safety factor of sliding mass can be computed precisely by using the rigorous limit equilibrium method. The initial normal stress distribution over the 3D slip surface was assumed, and then it was modified by a function with 2 parameters to satisfy two force equilibrium conditions about $x$ - and $z$-axis and one moment equilibrium condition around $y$-axis. An iterative equation was derived that it would yield a value to $3 \mathrm{D}$ safety factor. The values of the three-dimensional safety factor are computed with different assumption of initial normal stresses. The computation results show that the influence of assumption of the three-dimensional initial normal stress on the safety factor is negligible because the maximum different value of the safety factor of the symmetrical threedimensional slope is below $5 \%$. So the results are accurate that they can be directly applied to engineering.

\section{Data Availability}

The data used to support the findings of this study are available from the corresponding author upon request.

\section{Additional Points}

This paper mainly studies the influence of initial normal stress assumption of slip surface on safety factor of symmetrical three-dimensional slopes. By using the explicit solution of the three-dimensional slope stability based on the modification of normal stress distribution over the slip surface, the influence 
of assumption of the three-dimensional initial normal stress on the safety factor is investigated. The initial normal stress distribution over the 3D slip surface was assumed, and then it was modified by a function with 2 parameters to satisfy all forces and moment equilibrium conditions. The computation results show that the influence of assumption of the threedimensional initial normal stress on the safety factor of symmetrical three-dimensional slopes is negligible because the maximum different value of three-dimensional factor safety is below $5 \%$.

\section{Conflicts of Interest}

The author declares that there are no conflicts of interest.

\section{Acknowledgments}

This paper was funded by the National Natural Science Foundation of China (51508570).

\section{References}

[1] J. M. Duncan, "State of the art: limit equilibrium and finiteelement analysis of slopes," Journal of Geotechnical Engineering, vol. 122, no. 7, pp. 577-596, 1996.

[2] K. Ugai and D. Leshchinsky, "Three-dimensional limit equilibrium and finite element analyses: a comparison of results," Soils and Foundations, vol. 35, no. 4, pp. 1-7, 1995.

[3] K. Narita and H. Yamaguchi, "Three-dimensional bearing capacity analysis of foundations by use of a method of slices," Soils and Foundations, vol. 32, no. 4, pp. 143-155, 1992.

[4] D. Y. Zhu, H. L. Liu, P. X. Fan et al., "Explicit solution to threedimensional factor of safety of rotational symmetrical slope," Journal of PLA University of Science and Technology, vol. 7, no. 5, pp. 446-449, 2006.

[5] D. Y. Zhu, X. L. Ding, and J. H. Deng, "Explicit solution to 3D safety factor of slope based on force equilibrium and its application to engineering," Rock \& Soil Mechanics, vol. 29, no. 8, pp. 2011-2003, 2008.

[6] W. Fellenius, "Calculation of the stability of earth dams," in Proceedings of Transactions of the 2nd Congress on Large Dams, International Commission on Large Dams of the World Power Conference, vol. 4, pp. 445-462, Washington, DC, USA, December 1936.

[7] A. W. Bishop, "The use of the slip circle in the stability analysis of slopes," Géotechnique, vol. 5, no. 1, pp. 7-17, 1955.

[8] X. Zhang, "Three-dimensional stability analysis of concave slopes in plan view," Journal of Geotechnical Engineering, vol. 114, no. 6, pp. 658-671, 1988.

[9] O. Hungr, "An extension of Bishop's simplified method of slope stability analysis to three dimensionsfied method of slope stability analysis to three dimension," Géotechnique, vol. 37, no. 1, pp. 113-117, 1987.

[10] D. Leshchinsky, R. Baker, and M. L. Silver, "Three dimensional analysis of slope stability," International Journal for Numerical and Analytical Methods in Geomechanics, vol. 9, no. 3, pp. 199-223, 1985.

[11] M. M. Baligh and A. S. Azzouz, "End effects on stability of cohesive slopes," Journal of the Geotechnical Engineering Division, vol. 101, no. 11, pp. 1105-1117, 1975.

[12] A. Gens, J. N. Hutchinson, and S. Cavounidis, "Threedimensional analysis of slides in cohesive soils," Géotechnique, vol. 38, no. 1, pp. 1-23, 1988. 


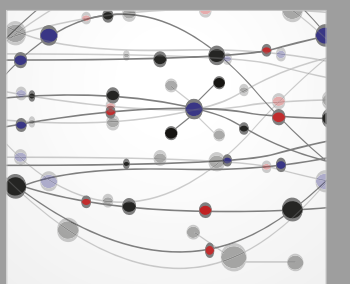

The Scientific World Journal
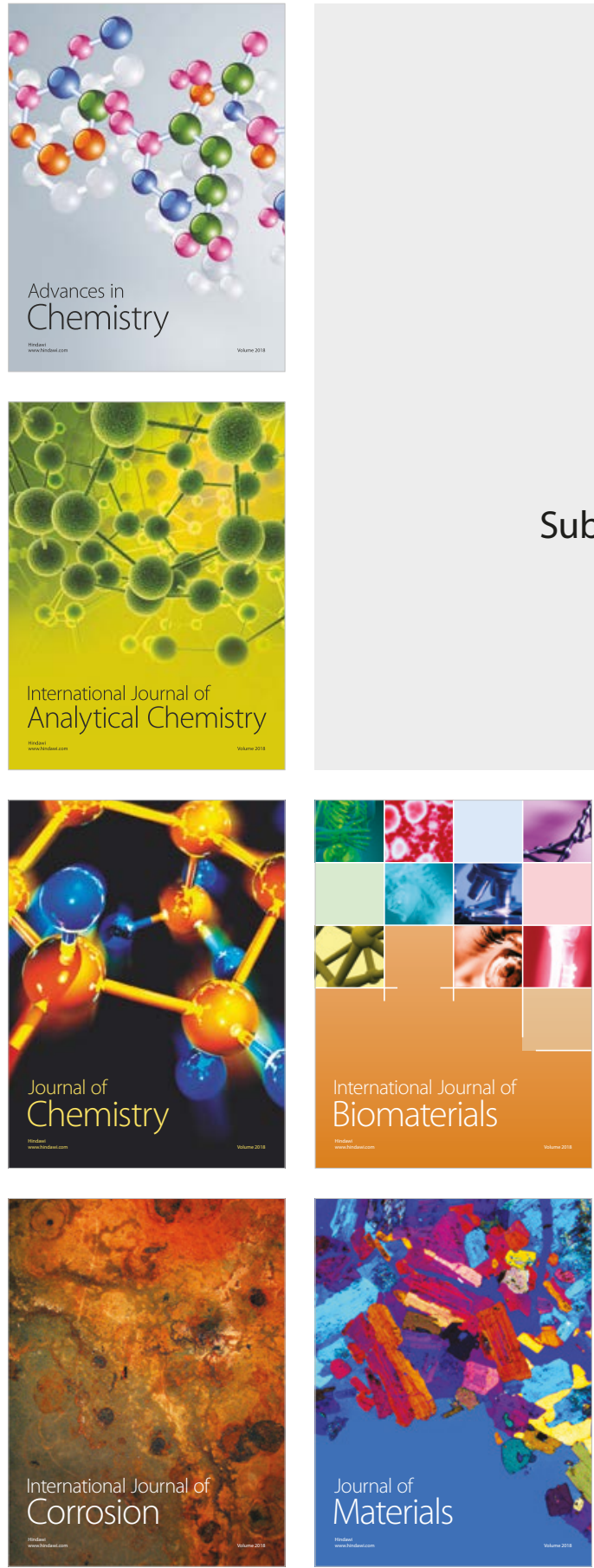

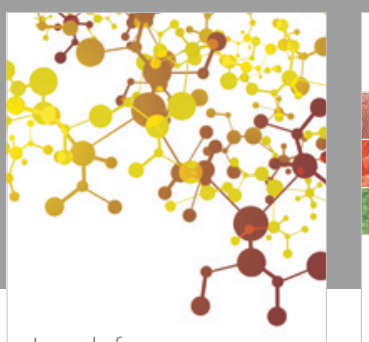

Journal of

Applied Chemistry
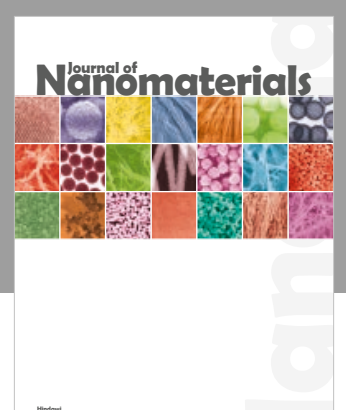

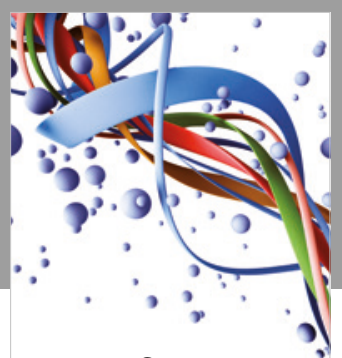

Scientifica

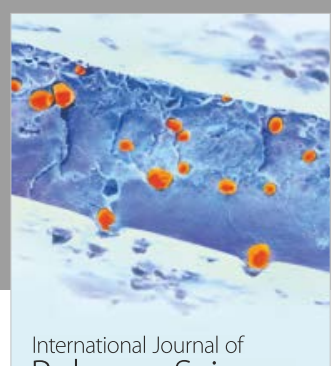

Polymer Science

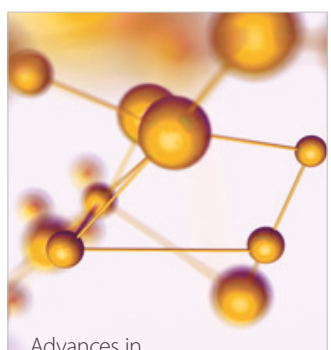

Physical Chemistry
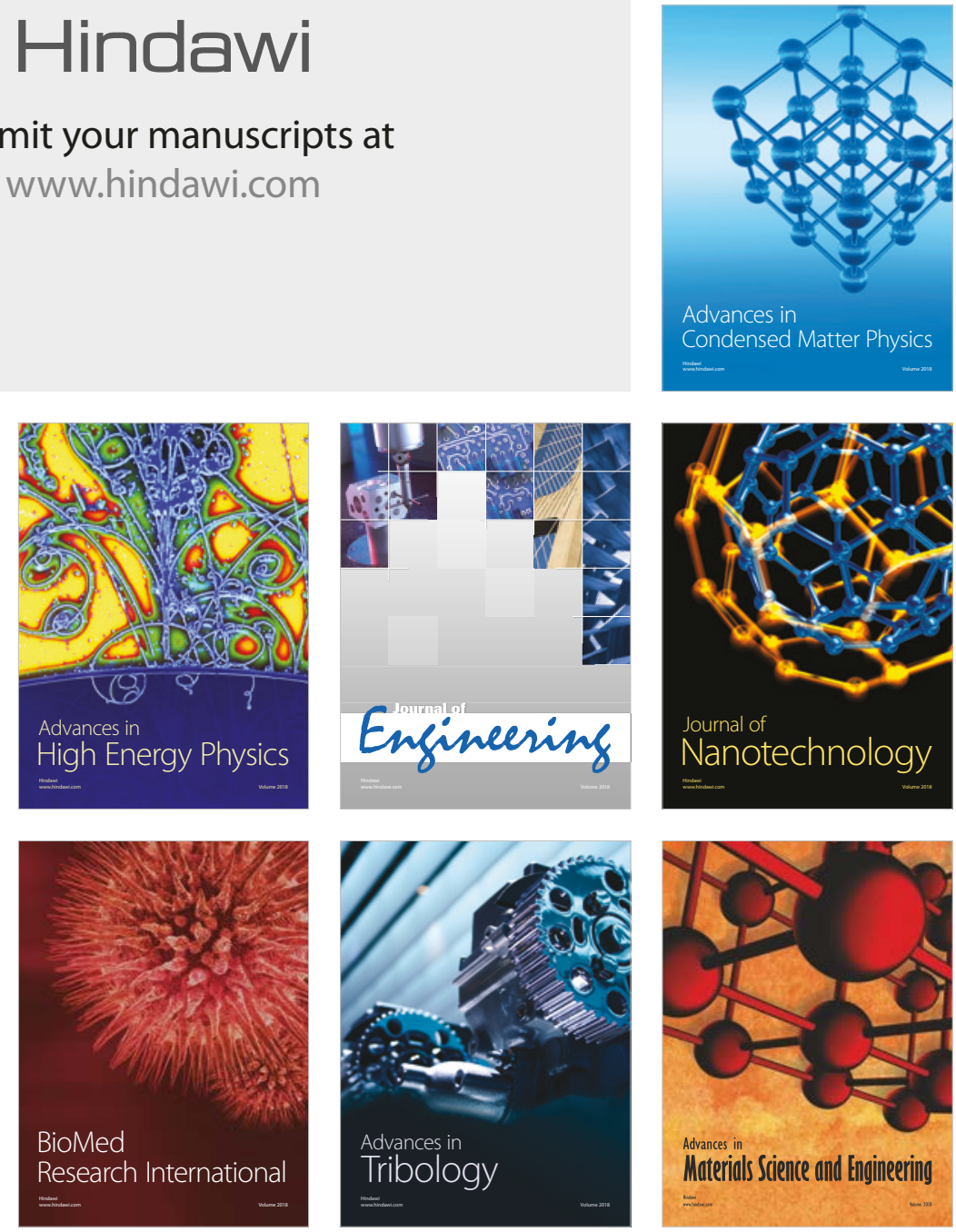